\title{
A STATE ESTIMATOR FOR LV NETWORKS: RESULTS FROM THE EVOLVDSO PROJECT
}

\author{
Henrique TEIXEIRA \\ INESC TEC - Portugal \\ henrique.s.teixeira@inesctec.pt \\ Ricardo BESSA \\ INESC TEC - Portugal \\ ricardo.j.bessa@inesctec.pt \\ Ana C. MORAIS \\ EDP Distribuição - Portugal \\ anacarina.morais@edp.pt
}

\author{
Pedro N. PEREIRA BARBEIRO \\ INESC TEC - Portugal \\ pedro.p.barbeiro@inesctec.pt
}

Pedro G. MATOS

EDP Distribuição - Portugal

pedro.godinhomatos@edp.pt

\author{
Mathieu CAUJOLLE \\ EDF - R\&D - France \\ mathieu.caujolle@edf.fr
}

\author{
Jorge PEREIRA \\ INESC TEC and FEP - Portugal \\ jpereira@inesctec.pt \\ Diogo LEMOS \\ EDP Distribuição - Portugal \\ diogo.lemos@edp.pt \\ Maria SEBASTIAN-VIANA \\ ERDF - France \\ maria.sebastian-viana@erdf.fr
}

\begin{abstract}
The increasing connection of new assets in LV networks will surely require a better monitoring of these systems in a real-time manner. In order to meet this purpose, a Distribution State Estimator (DSE) module clearly appears as the most cost-effective and possibly the only reliable option available. In this sense, in the scope of the evolvDSO project, a DSE tool exploiting the concept of ELM-AE was developed and tested in two distinct real $L V$ distribution networks. In this paper the main results achieved with the proposed DSE tool are presented and discussed.
\end{abstract}

\section{INTRODUCTION}

The increase of distributed generation units and new equipment such as electric vehicles and energy storage devices connected to distribution networks, particularly at the Low Voltage (LV) level, is demanding for further monitoring of such systems. However, monitoring all grid points in real-time will be for sure impracticable due to the expected cost of such metering devices together with the associated communication costs. Thus, a Distribution State Estimator (DSE) module is going to play an important role in the operation of the future distribution systems.

In contrast with transmission networks, distribution ones are poorly monitored and, additionally, their technical parameters and topology may be completely or partially unknown in particular for the LV networks. In this sense, the direct application of the conventional state estimation algorithms (e.g. based on weighted least squares methods) is not possible. However, with the emergence and development of Smart Grids in the near future, it is reasonable to think that each consumer and/or producer connected to LV networks will have installed at its premise a Smart Meter (SM) capable of monitoring several electrical quantities (voltage magnitude as well as active and reactive power values) and transmitting them to a database server periodically (for instance, once a day). This electrical information can then be used to feed a state estimation tool, which can run either centralized, if located at the Distribution Management System level, or dispersed, if located at the Distribution Transformer Controller (DTC) level of the correspondent MV/LV substation.

A non-conventional DSE tool capable of taking advantage of historical information stored in a database and use it to estimate the state of the system in poorly characterized three-phase LV systems was proposed in [1]. This DSE tool relies on a specific type of artificial neural networks the Autoencoders (AE) - trained with a resilient backpropagation algorithm and then applies a meta-heuristic procedure (an Evolutionary Particle Swarm Optimization algorithm - EPSO) to find the system state variables.

The evolvDSO project aims at defining the future roles of Distribution System Operators and the development of tools to fulfil such roles, taking into account the growing relevance of distributed renewable energy sources in the generation mix and the increasingly pro-active demand for electricity. In the scope of the evolvDSO project, a DSE tool based on the methodology presented in [1], but employing a completely different concept for training the $\mathrm{AE}$, the concept of Extreme Learning Machine Autoencoder (ELM-AE) [2, 3], was developed. Applying this kind of ELM techniques [4] to properly train an AE was intended to enhance the estimation accuracy as well as to reduce the training and running times of the DSE. In [2, 3] the methodology behind the DSE tool developed within the evolvDSO project was already presented. At that time, the tool's performance was evaluated using a simulated historical database in a small typical Portuguese LV network of 33 nodes [3]. In this paper are now presented some of the main results achieved in the evolvDSO project regarding the tool's validation in real LV distribution networks and using real historical data gathered from SM installed in the field.

\section{METHODOLOGY AND STUDY CASES}

As mentioned before, the DSE tool developed in the context of the evolvDSO project is based on the use of $\mathrm{AE}$ trained through ELM techniques. A detailed description of the overall methodology, mainly regarding the training algorithm, may be found in [3]. Nevertheless, it is important to bear in mind that an historical database with all data temporally synchronized is required so that the $\mathrm{AE}$ can effectively learn the patterns/correlations between the electrical variables of a given network, and thus perform a reliable state estimation. The database needs to contain data about the variables that will be passed to the $\mathrm{AE}$ during its training stage, which includes the missing signals and the measurements that will be available in realtime. 
After having properly trained the ELM-AE, the measurements available in real-time are used as its inputs to guide the optimization algorithm towards the state estimation solution. The solution comprises the state variables to be estimated: voltage magnitude values, as well as the active power and reactive power injections if desired. Figure 1 presents the main steps involved in this estimation process.

This methodology was evaluated for two distinct real LV distribution networks, one from Portugal and the other from France.

\section{Portuguese LV network}

The Portuguese LV system is a rural network that is part of a Smart Grid Pilot Site developed under the InovGrid project. It has two main feeders which are connected to a MV/LV secondary substation $(30 \mathrm{kV} / 400 \mathrm{~V} / 230 \mathrm{~V})$ equipped with a transformer of $100 \mathrm{kVA}$ rated power. The network is composed by overhead lines with a high $\mathrm{R} / \mathrm{X}$ ratio (around 10 in average) and has a total of 74 nodes containing 44 customers (42 single-phase customers with contracted powers between 1.15 and $6.9 \mathrm{kVA}$ and 2 threephase customers with contracted powers of 10.35 and $17.25 \mathrm{kVA}$ ). There are no microgeneration units installed in this network. Each customer connected to this network owns a SM capable of monitoring synchronously its active and reactive power consumption and voltage magnitude values.

\section{Historical database}

The historical database includes real average records related to the load (in time steps of 15 minutes) of the active and reactive power values, as well as voltage magnitude values for a period approximately of two months and a half (a universe of 7456 samples). About 6784 samples of the historical database were selected for training purposes and the other 672 samples for the evaluation set.

\section{Number of smart meters with real-time capabilities}

In order to test the presented methodology, two scenarios considering a different number of SM with the capability of transmitting data in real-time $\left(\mathrm{SM}_{\mathrm{r}}\right)$ were assumed.

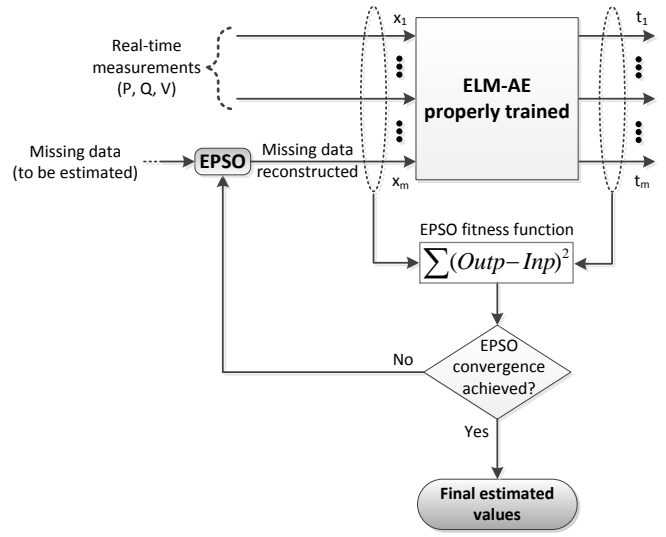

Figure 1: ELM-AE methodology illustration.
Table I: Real-time measurements scenarios for Portugal

\begin{tabular}{|c|c|c|c|}
\hline Scenario & $\begin{array}{c}\text { No. of } \\
\mathbf{S M}_{\mathbf{r}}\end{array}$ & $\begin{array}{c}\text { No. of real-time } \\
\text { measurements }\end{array}$ & $\begin{array}{c}\text { No. of variables to } \\
\text { be estimated }\end{array}$ \\
\hline $\mathbf{1}$ & 6 & 23 & 36 \\
\hline $\mathbf{2}$ & 12 & 41 & 30 \\
\hline
\end{tabular}

Scenario 1, where $6 \mathrm{SM}_{\mathrm{r}}$ are considered, represents the real network field situation, i.e., these $\mathrm{SM}_{\mathrm{r}}$ are the ones installed in the field. It is important to state that all of these $\mathrm{SM}_{\mathrm{r}}$ were installed only in one of the two feeders (equally distributed between phases) due to some technical constraints. Thus, only one feeder in this scenario was monitored in real-time.

A more optimistic scenario was also considered for comparison purposes. In scenario 2, $12 \mathrm{SM}_{\mathrm{r}}$ equally distributed between all phases of both feeders ( $2 \mathrm{SM}_{\mathrm{r}}$ per phase in each feeder) were assumed. This criterion was taken to ensure the existence of electrical information regarding all phases of each feeder. In this scenario both feeders were real-time monitored and a study was carried out to find the most suitable locations (customers' premise) to place a $\mathrm{SM}_{\mathrm{r}}$ by doing a correlation analysis between the voltage magnitude values available in the historical database.

Table I summarizes information about the scenarios considered in the Portuguese case study.

\section{French LV network}

The French LV network is composed by underground and overhead lines with a moderated $\mathrm{R} / \mathrm{X}$ ratio (1.7 in average). It is connected to a MV/LV secondary substation equipped with a $20 \mathrm{kV} / 400 \mathrm{~V}(230 \mathrm{~V})$ transformer with a rated power of $400 \mathrm{kVA}$. The network has a total of 77 nodes containing 132 single-phase customers with contracted powers varying between 3 and $12 \mathrm{kVA}$ and 3 three-phase customers with contracted powers of 12 and $18 \mathrm{kVA}$. This network also contains 14 microgeneration units (photovoltaic panels), of which 12 are single-phase connected with installed capacities between 2.9 and 18 $\mathrm{kW}$ and 2 units are three-phase, correspondently with 8 and $18 \mathrm{~kW}$ of installed capacity. For this case study, it was assumed that each customer had a SM capable of monitoring synchronously (in time steps of 10 minutes) the active power and the voltage magnitude values.

\section{Historical database}

Differently from the Portuguese network, no telemetry data regarding the bus voltage magnitudes and power measurements in loads and microgeneration units was available for this case study. Therefore, these data were generated through a three-phase power flow algorithm based on backward/forward sweep method and using a small real historical database from another French LV network as the starting point. Six months of simulated data were then generated. The historical database was divided in two sets: the last week (1008 samples) was used as the evaluation set and the remaining (25344 samples) was used for training purposes. 
Table II: Real-time measurements scenarios for France

\begin{tabular}{|c|c|c|c|}
\hline Scenario & $\begin{array}{c}\text { No. of } \\
\mathbf{S M}_{\mathbf{r}}\end{array}$ & $\begin{array}{c}\text { No. of real-time } \\
\text { measurements }\end{array}$ & $\begin{array}{c}\text { No. of variables to } \\
\text { be estimated }\end{array}$ \\
\hline $\mathbf{1}$ & 5 & 24 & 150 \\
\hline $\mathbf{2}$ & 10 & 38 & 143 \\
\hline $\mathbf{3}$ & 15 & 48 & 138 \\
\hline $\mathbf{4}$ & 20 & 58 & 133 \\
\hline
\end{tabular}

Number of smart meters with real-time capabilities In the French LV network, the approach followed to determine the number of $\mathrm{SM}_{\mathrm{r}}$ consisted in iteratively replacing the existing $\mathrm{SM}$ by $\mathrm{SM}_{\mathrm{r}}$ in the nodes with the largest Mean Absolute Error (MAE) until an acceptable value for the MAE was obtained. Whenever a given network node had several SM (consumers and/or producers) connected to, the SM that led to a lower MAE was the only one replaced by a $\mathrm{SM}_{\mathrm{r}}$.

Twenty $\mathrm{SM}_{\mathrm{r}}$ were required to meet the established MAE target. Four scenarios with different number of $\mathrm{SM}_{\mathrm{r}}$ were considered. These scenarios are presented in Table II together with information regarding the number of realtime measurements and variables to be estimated.

In both study cases it was considered that the MV/LV secondary substation held a DTC which can, among other functions, concentrate the SM information - like an headend server. Furthermore, the associated measurement equipment with the capability of monitoring in real-time the active and reactive power flows in the transformer (when available) and the voltage magnitude (three values, one per phase) at the LV side of the transformer was also assumed. Such measurements were taken into account in the calculation of the real-time measurements number shown in Table I and Table II.

\section{RESULTS}

The results presented in this section are referred to the entire evaluation sets previously defined.

\section{Portuguese LV network}

In Figure 2, the boxplots depict the absolute error obtained in scenarios 1 and 2 for the voltage magnitude in all customers' premises not being monitored in real-time. The absolute error was calculated between the real values (gathered by the SM installed at customers' premise) and the estimated values obtained with the DSE algorithm. As it was expected, the estimation accuracy is improved when more real-time measurements are available. However, the improvement verified in scenario 2 , comparing with scenario 1 , was not only due to the number of real-time measurements available (twice the number of scenario 1 ), but also to their location, which contributed to overcome the lack of electrical information in both network feeders. Regarding this, it should be recalled that in scenario 1 only one of the two substation feeders has customers with $\mathrm{SM}_{\mathrm{r}}$, whereas in scenario 2 both feeders have the same number of customers with $\mathrm{SM}_{\mathrm{r}}$.

The state estimation error obtained in scenario 1 accounts for the worst results. Nevertheless, the estimation error is lower than $4 \mathrm{~V}$ in $75 \%$ of the samples analyzed in the large majority of the SM. In what concerns to scenario 2 , this error is lower than $1.08 \mathrm{~V}$ in $75 \%$ of the samples analyzed. The results achieved give good indications regarding the estimation accuracy of the DSE. This fact becomes more relevant taking into account that in scenarios 1 and 2 only $14 \%$ and $27 \%$ of the customers own a $\mathrm{SM}_{\mathrm{r}}$, respectively. The MAE obtained for scenarios 1 and 2 was correspondingly $1.77 \mathrm{~V}$ and $0.49 \mathrm{~V}$.

\section{French LV network}

Due to space restrictions and the number of customers present in this network, only the results regarding the customers connected to phase A are shown for the scenarios 1 and 4 . The results obtained for the other two phases are analogous. In Figure 3 the voltage magnitude absolute error for all customers (not being monitored in real-time) connected to phase A is presented.
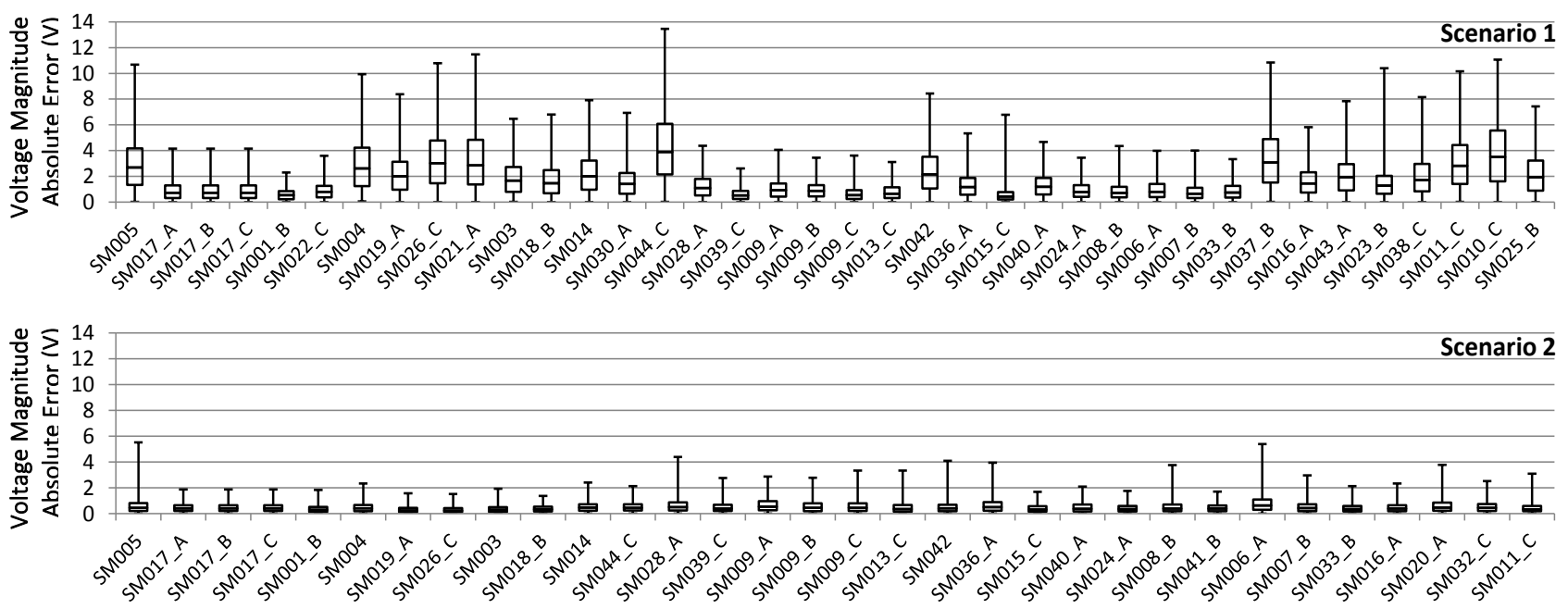

Figure 2: Voltage magnitude absolute error for all customers connected to the Portuguese LV network. 

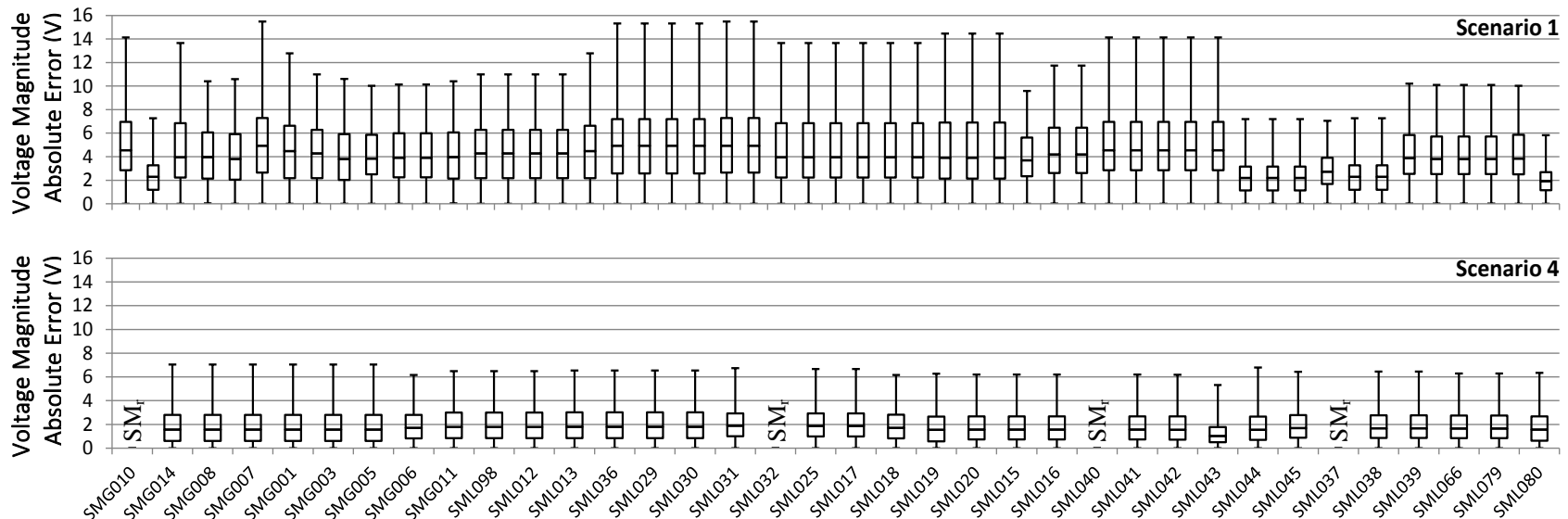

Figure 3: Voltage magnitude absolute error for all customers connected to phase A of the French LV network.

As the historical database for the French LV network consisted in simulated data, the real values for the absolute error calculation were the values generated through power flows. Similarly to the results presented for the Portuguese case, there is a clear general improvement in the state estimation results when more measurements are available in real-time. In fact, in the scenario 4 , the voltage magnitude absolute error obtained is lower than $4 \mathrm{~V}$ in $75 \%$ of the samples analyzed for all the customers connected to each phase. The maximum MAE obtained for scenario 4 was $2.3 \mathrm{~V}$, a value verified for phase $\mathrm{C}$ (Table III). It should be remembered that in this scenario, only $13 \%$ of the total customers own a $\mathrm{SM}_{\mathrm{r}}$, which can be seen as a good indicator regarding the accuracy of the proposed DSE for the estimation of voltage magnitude values.

\section{CONCLUSION}

The DSE developed in the scope of the evolvDSO project had already been evaluated in a typical LV network using a simulated historical database [3]. In this paper the results for two distinct real $\mathrm{LV}$ distribution networks and using real metering data were presented. The results confirm the conclusions already attained with simulated data, i.e., the good performance of the DSE tool regarding the estimation of voltage magnitude values. In fact, the results demonstrated that the estimation of voltage magnitude values can be very effective and accurate when using an ELM-AE properly trained. This feature is preserved even when real data are used and a low number of real-time measurements are available. As expected, adding real-time measurements leads to a general improvement of the state estimation accuracy. However, the trade-off between a better accuracy and an increased cost should be carefully analyzed.

Finally, it should be pointed out that one of the main advantages of this tool is its capability to achieve a state estimation solution without any knowledge of the networks' parameters and topology, what would be impossible with the traditional state estimation techniques.
Table III: Voltage magnitude MAE obtained.

\begin{tabular}{|c|c|c|c|}
\hline Scenario & Phase A $(\mathbf{V})$ & Phase B $(\mathbf{V})$ & Phase C $(\mathbf{V})$ \\
\hline $\mathbf{1}$ & 4.2 & 3.4 & 4.0 \\
\hline $\mathbf{2}$ & 3.5 & 2.7 & 3.4 \\
\hline $\mathbf{3}$ & 2.3 & 2.1 & 3.1 \\
\hline $\mathbf{4}$ & 2.2 & 1.9 & 2.3 \\
\hline
\end{tabular}

Also, the short computation time required for both training and state estimation procedures needs to be emphasized as another important advantage over traditional techniques.

\section{ACKNOWLEDGEMENTS}

The research leading to this work is being carried out as a part of the evolvDSO project (Development of methodologies and tools for new and evolving DSO roles for efficient DRES integration in distribution networks) www.evolvdso.eu. This project is funded by the European Commission under the seventh framework program (FP7) under grant agreement number 608732 .

\section{REFERENCES}

[1] P. N. P. Barbeiro, H. Teixeira, J. Krstulovic, J. Pereira, and F. J. Soares, 2015, "Exploiting autoencoders for three-phase state estimation in unbalanced distributions grids", Electric Power Systems Research, vol. 123, 108-118.

[2] P. N. P. Barbeiro, H. Teixeira, J. Pereira, and R. Bessa, 2015, "An ELM-AE State Estimator for RealTime Monitoring in Poorly Characterized Distribution Networks", in Proc. IEEE Eindhoven PowerTech, 1-6.

[3] H. Teixeira, P. N. P. Barbeiro, J. Pereira, R. Bessa, and et al., 2015, Advanced Tools and Methodologies for Forecasting, Operational Scheduling and Grid Optimisation, Deliverable D3.2 of the evolvDSO Project.

[4] G.-B. Huang, Q.-Y. Zhu, and C.-K. Siew, Dec. 2006, "Extreme learning machine: Theory and applications", Neurocomputing, vol. 70, no. 1-3, 489501. 